\title{
Gastric cavernous hemangioma: A rare case with upper gastrointestinal bleeding
}

\author{
LIANG ZONG, PING CHEN, GUO HAO SHI and LEI WANG \\ Department of Gastrointestinal Surgery, Su Bei People's Hospital of Jiangsu Province, \\ Yangzhou University, Yangzhou, Jiangsu 225001, P.R. China
}

Received March 22, 2011; Accepted June 30, 2011

DOI: $10.3892 / \mathrm{ol} .2011 .364$

\begin{abstract}
Upper gastrointestinal bleeding caused by a gastric hemangioma is a relatively rare occurrence. We report the case of a 65-year-old woman who suffered a sudden onset of hematemesis. Endoscopy revealed a $4 \times 3 \mathrm{~cm}$ mass located in the gastric fundus. Abdominal contrast-enhanced CT revealed the shadow of enhancing linear blood vessels located in the gastric fundus. Based on her clinical appearance and the laboratory results, the patient was diagnosed with gastric hemangioma. In the laparotomy, a proximal gastrectomy was performed. The final diagnosis of cavernous hemangioma arising from the gastric fundus was confirmed by postoperative pathological examination.
\end{abstract}

\section{Introduction}

Hemangioma appear in various parts of the human body, including the liver, spleen, colon, retroperitoneum, adrenal glands, soft tissues, extremities, central nervous system and mediastinum (1). Gastric hemangioma, a rare tumor occurring mostly in the antrum, was first described by Lammers in 1893 (2). It represents approximately $1.7 \%$ of all gastric benign tumors and $20 \%$ of unknown hemorrhage. In this case report, we present the clinical presentation, diagnosis and treatment of a cavernous hemangioma that arises from the gastric fundus.

\section{Case report}

The patient is a 65-year-old Chinese female who suffered a sudden onset of hematemesis with approximately $800 \mathrm{ml}$ blood loss in a total of $10 \mathrm{~h}$. When she was admitted to hospital, she was in a state of pre-shock with the complaint of muscle weakness and dizziness. She had no past history of diabetes, hypertension, hepatitis or bronchial asthma. The patient did not

Correspondence to: Professor Ping Chen, Gastrointestinal Surgery Department, Su Bei People's Hospital of Jiangsu Province, Yangzhou University, Yangzhou, Jiangsu 225001, P.R. China E-mail: chen86ky@126.com

Key words: gastric tumor, cavernous hemangioma, upper gastrointestinal bleeding take any medication, including over-the-counter non-steroidal anti-inflammatory drugs (NSAIDs), and was not taking herbal supplements. On abdominal examination she felt discomfort only in the upper abdomen, without tenderness, and she was hemodynamically unstable. Routine blood tests, including hepatic and renal function tests, revealed a hemoglobin level of $56 \mathrm{~g} / 1$, a platelet count of $93 \times 10^{9} / 1$, elevated blood urea nitrogen (BUN) of $10.25 \mathrm{mmol} / 1$, total protein of $53.9 \mathrm{~g} / \mathrm{l}$, albumin of $30.8 \mathrm{~g} / 1$, serum creatinine of $60.9 \mu \mathrm{mol} / 1$, lactate dehydrogenase (LDH) of $199 \mathrm{U} / 1$, uric acid of $121.0 \mu \mathrm{mol} / 1$, and an erythrocyte sedimentation rate (ESR) of $31 \mathrm{~mm} / \mathrm{h}$. Based on the patient's clinical appearance and the laboratory analysis, she was diagnosed with acute upper gastrointestinal bleeding and hemorrhagic shock.

An urgent endoscopy following active resuscitation revealed a $4 \times 3 \mathrm{~cm}$ mass located in the gastric fundus with active hemorrhage at the surface (Fig. 1A). The bleeding was controlled

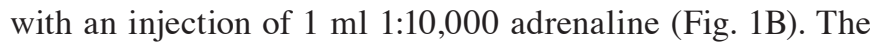
patient underwent prompt treatment with vigorous intravenous (IV) rehydration, blood transfusion for worsening anemia, and IV ranitidine therapy. Following stabilization, abdominal computed tomography with its periphery enhanced by the contrast material in the delayed phase revealed the shadow of enhancing linear blood vessels located in the gastric fundus (Fig. 1). No abnormalities were revealed in the laboratory data of the tumor markers, which are listed as: CA19-9, $6.39 \mathrm{KU} / 1$ (<35.00); CA242, 3.41 KU/1 (<20.00); CA125, $2.27 \mathrm{KU} / 1$ (<35.00); CA15-3,2.28 KU/l(<35.00); NSE, <1.0 ng/ml(<13.00); CEA, $3.25 \mathrm{ng} / \mathrm{ml}(<5.00)$; ferritin, $20.10 \mathrm{ng} / \mathrm{ml}$ (male $<322.00$, female<219.00); $\beta$-HCG, <0.03 MIU/ml (<3.00); AFP, $5.68 \mathrm{ng} /$ $\mathrm{ml}(<20.00) ; \mathrm{HGH}, 2.00 \mathrm{ng} / \mathrm{ml}(<7.50)$. In the laparotomy, a soft mass located in the gastric fundus was found to vibrate when pressure of the hand was applied. A proximal gastrectomy was performed. The postoperative period was uneventful and the patient was discharged 10 days after the surgery. The final diagnosis of cavernous hemangioma arising from the gastric fundus was confirmed by postoperative pathological examination.

\section{Discussion}

Kaijser has classified gastrointestinal hemangioma pathologically as multiple phlebectasia, cavernous hemangioma, capillary hemangioma and angiomatosis (3). 


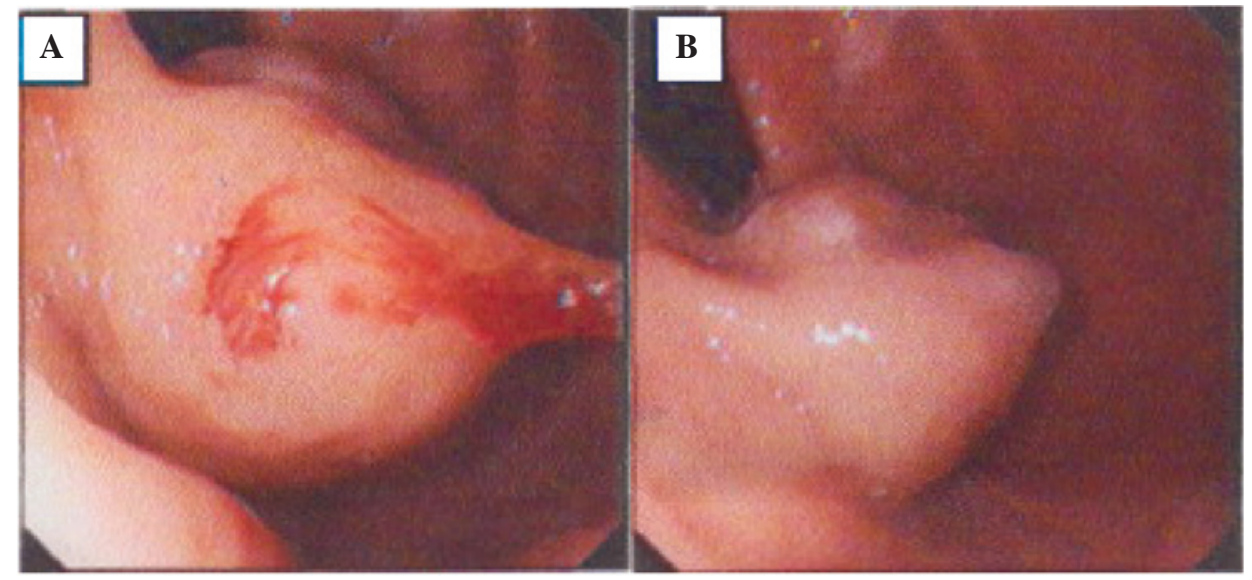

Figure 1. (A) Emergency endoscopy revealed a mass located in the gastric fundus with active hemorrhaging at the surface. (B) The bleeding is controlled by injecting 1:10,000 adrenaline.

A

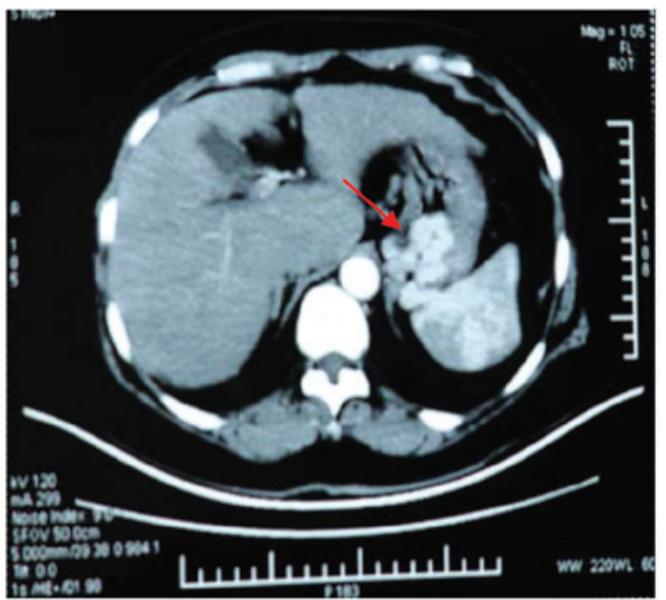

B

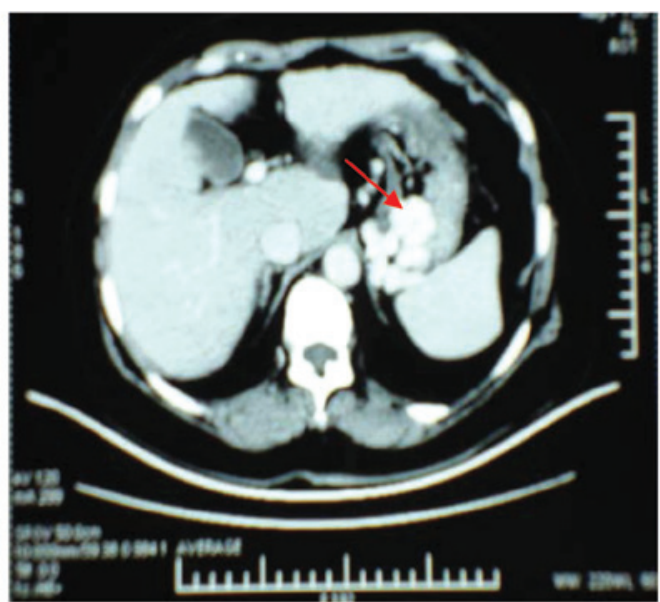

Figure 2. (A) Computed tomography showing a circuitous and nodal shadow located in the gastric fundus. (B) Contrast-enhanced computed tomography showing the shadow of enhancing linear blood vessels.

A

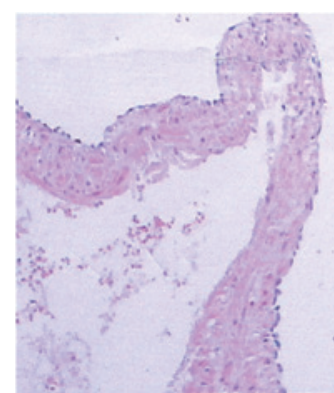

HE: $10 * 10$
B

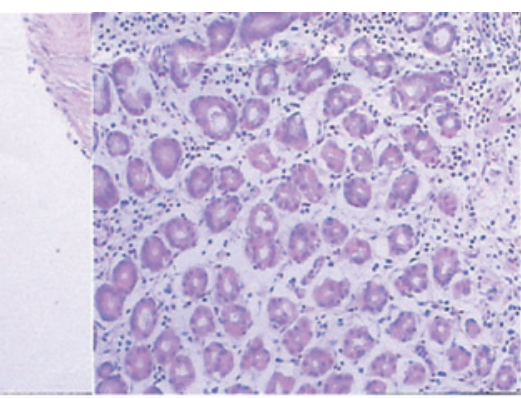

HE: $10 * 10$

Figure 3. Pathological section with H\&E staining showing (A) large tortuous arterial vessels located in the submucosa with simple squamous epithelium in the lining of lumens. (B) Surrounding normal gastric tissue.

Abdominal cavernous hemangiomas usually originate in the liver, but occasionally present in the stomach with the same origin and mechanism as other cavernous angiomas, which have been considered as a congenital, benign and abnormal development. Findings of previous studies have shown that

cavernous hemangiomas are congenital hamartomatous lesions that originate from the mesodermal remnant tissues. These hemangiomas are composed of large dilated blood vessels and contain large blood-filled spaces that are caused by dilation and thickening of the walls of the capillary loops (4). Due to the thin walls of blood vessels, gastric cavernous hemangiomas $(\mathrm{GCH})$ are prone to rupture with rapid blood flow. In this case report, we highlight the rarity of this lesion and the difficulties in diagnosing it preoperatively. Most gastrointestinal tract hemangiomas are of the cavernous type and upper gastrointestinal bleeding is the most common symptom (5). Gastric hemorrhages are common clinical emergencies, which often directly involve the surgeon in diagnosis and treatment; among these, rare cavernous hemangiomas deserve particular attention.

Endoscopy may serve to establish the diagnosis in those cases where, due to the hemangioma's small size or unfavorable location, radiological examination fails to detect them (2). Usually, emergency endoscopy is a means of diagnosis and treatment, but this was not effective in the present case since the delicate tissue tended to bleed. Furthermore, it is possible that this characteristic did not allow a correct tissue sample to be collected for pathological examination, which yielded 
a negative result. Radiological examination suggests the possible diagnosis, and the best definitive diagnostic procedure is CT scanning and MRI, which demonstrate the location and relationship of the lesion to neighboring structures as the preoperative reference of resectability. The lesion appears either as enhancing linear blood vessels or caputmedusae, a radial orientation of small vessels that resemble the hair of Medusa from Greek mythology. However, when the blood vessel signal is weak, CT scanning and MRI are incapable of distinguishing $\mathrm{GCH}$ from mesochymal tumors. However, $\mathrm{GCH}$ may be misdiagnosed as a stromal tumor. Surgical treatment is a definitive modality, and recurrence following complete resection has not been reported thus far. The final diagnosis in the present case was obtained by definitive histopathology.

In conclusion, we report a rare case of cavernous hemangioma originating from the gastric fundus. It may cause diagnostic difficulties preoperatively as biopsy is not an option due to the submucosal location of the tumor and hemorrhagic factor. However, in the present case, we were able to obtain the result of enhancing linear blood vessels by contrast-enhanced CT scanning prior to the surgery, which assisted us in making a primary diagnosis. Therefore, abdominal images should be examined on a regular basis. Although this disease is benign with a lower recurrence following total resection, we nevertheless suggest the requirement for long-term follow-up to assess treatment outcome.

\section{Acknowledgements}

L. Zong and P. Chen performed the majority of this study and wrote the manuscript. G.H. Shi and L. Wang provided the collection of material from the database.

\section{References}

1. Kinoshita T, Naganuma $\mathrm{H}$ and Yajima Y: Venous hemangioma of the mesocolon. AJR Am J Roentgenol 169: 600-601, 1997.

2. Bongiovi JJ, Dufly JL and Healdow E: Gastric hemangioma associated with upper gastrointestinal bleeding. Arch Surg 95:93-95, 1967.

3. Kaijser R: Diagnosis of cavernous hemangiomas in the digestive tract. Acta Radio 22: 665-686, 1941.

4. Boley SJ, Sammartamo R, Adams A, et al: On the nature and etiology of vascular ectasias of the colon. Gastroenterology 72: 650-652, 1987.

5. Wan YL, Eng HL, Lee TY, et al: Computed tomography of an exophytic gastric hemangioma with torsion and intratumoral hemorrhage. Clin Imaging 17: 210-212, 1993. 